\title{
Differential Interference Suppression Aided Three-Stage Concatenated Successive Relaying
}

\author{
Li Li, Li Wang, Member, IEEE, and Lajos Hanzo, Fellow, IEEE
}

\begin{abstract}
Conventional single-relay aided two-phase cooperative networks employing coherent detection algorithms incur a significant $50 \%$ throughput loss. Furthermore, it is unrealistic to expect that in addition to the task of relaying, the relay-station would dedicate further precious resources to the estimation of the source-to-relay channel in support of coherent detection, which would consume extra energy expended in power-hungry channel estimation. In order to circumvent these problems, we propose successive relaying employing noncoherent detection schemes. A crucial challenge in this context is that of suppressing the successive relaying induced interference, despite dispensing with any channel state information (CSI). We overcome this challenge by introducing a novel adaptive Newton algorithm based multiple-symbol differential interference suppression (MSDIS) scheme. We demonstrate that our system is capable of nearerror-free transmissions at low signal-to-noise ratios.
\end{abstract}

Index Terms - Relaying, non-coherent detection, three-stage concatenated iterative detection, interference suppression.

\section{INTRODUCTION}

C OHERENT detection schemes impose a substantial energy consumption owing to complex and powerhungry channel estimation, which may become prohibitive in Multiple-Input Multiple-Output (MIMO) systems, where numerous channels have to be estimated. Consequently, noncoherent detection schemes dispensing with channel estimation become an attractive low-complexity alternative. The multiplesymbol differential detection (MSDD) algorithm was characterized by Ho and Fung in [1]. Then Lampe et al. devised the multiple-symbol differential sphere detection (MSDSD) algorithm by combing sphere detection with MSDD [2] and further developed it for conceiving its soft-input soft-output MSDSD (SISO-MSDSD) version in [3]. Recently, MSDSD was incorporated in cooperative noncoherent detection-based, relay-aided systems [4], [5].

Due to the limited antenna-separation of shirt-pocket-sized mobiles, the spatial diversity gain promised by MIMO systems [6] was shown to be eroded. By contrast, the family of cooperative techniques heralded by Van Der Meulen [7] is capable of achieving uplink transmit diversity by forming a virtual antenna array (VAA) in a distributed fashion. However, the conventional two-phase cooperative system incurs a severe

Paper approved by G. Bauch, the Editor for MIMO, Coding and Relaying of the IEEE Communications Society. Manuscript received August 22, 2011; revised January 10, 2012.

The authors are with the School of ECS, University of Southampton, SO17 1BJ, United Kingdom (e-mail: \{115e08, lw05r, lh $\} @$ ecs.soton.ac.uk).

The financial support of the European Union under the auspices of the Concerto project, as well as that of the RC-UK under the auspices of the India-UK Advanced Technology Centre known as In-ATC and of the ChinaUK Science Bridge is gratefully acknowledged.

Digital Object Identifier 10.1109/TCOMM.2012.051412.110557 multiplexing loss due to the half-duplex constraint of practical transceivers. In order to recover the resultant $50 \%$ throughput loss, the successive relaying aided network (SRAN) concept was proposed by Fan et al. in [8]. Nevertheless, the interference encountered both at the relays and at the destination significantly degrade the benefits of the successive relaying regime.

The inter-relay interference (IRI) problem of SRAN had been considered in [9], [10]. Regretfully, the interference suppression schemes advocated in these contributions were all based on the assumption that the receiver perfectly knows the CSI of all the links involved. Hence, the implementation of interference suppression for the SRAN remains a challenge, when employing noncoherent detection. Additionally, the differential interference suppression (DIS) philosophy was discussed in [11] and further developed in [12], where a novel amalgam of the adaptive modified Newton algorithm of [13] and of SISO-MSDSD was created.

Hence, our main contributions in this paper are:

1) We conceive successive relaying induced interference suppression at the destination, which is achieved despite dispensing with CSI by incorporating multiplesymbol differential interference suppression (MS-DIS) in SRANs.

2) Consequently, a new adaptive MS-DIS filter and MSDSD decoder assisted channel-code-aided three-stage turbo decoder is designed for the Base Station's (BS) receiver. We demonstrate that our proposed transceiver is capable of efficiently suppressing the interference by imposing as little as $2 \%$ training overhead, despite experiencing severe time-selective Rayleigh fading.

The rest of this paper is organised as follows. Our system model is portrayed in Section II. In Section III, the relevant concept of the adaptive MS-DIS scheme is introduced. We design the architecture of the transceiver in Section IV. The noncoherent Discrete-input Continuous-output Memoryless Channel (DCMC) capacity of our proposed system is derived in Section V. The performance of the proposed transceiver is characterized in Section VI. Finally, we conclude in Section VII.

\section{System Model}

The classic transmission procedure of the SRAN is illustrated in Figure 1, where three consecutive phases are exhibited. Typically, in the even phase, the Mobile Station (MS) $s$ broadcasts its information stream, while the activated relay $r_{0}$ listens to $s$ and another activated relay $r_{1}$ decodes, re-encodes as well as forwards the signals received from $s$ during the 

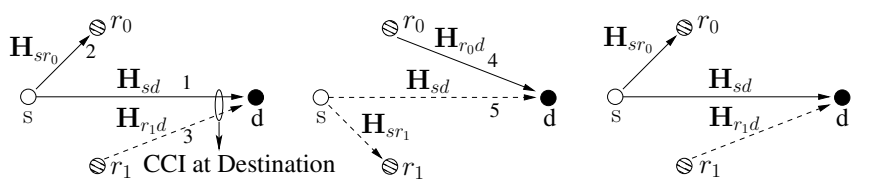

(a) Even Phase (or Frame)

(b) Odd Phase (or Frame)

(c) Even Phase (or Frame)

Fig. 1: Transmission processes of SRAN, different typical phases are shown.

most recent phase. In the consecutive odd phase, $s$ continues to broadcast its next information stream, but the roles of the relays $r_{0}$ and $r_{1}$ alternate $^{1}$. We assume that the resource allocation activates relays that are sufficiently far apart. Hence the interference between the relays is temporarily ignored. Furthermore, we assume that all the channels involved in the transmissions are narrowband time-selective Rayleigh fading channels and the notation $\mathbf{H}_{a b},(a, b) \in\left\{s, r_{0}, r_{1}, d\right\}$ represents the channel impulse response (CIR) matrix spanning from entity $a$ to $b$. Furthermore, the relay-aided path-loss reduction [15] is also considered in our system. Accordingly, the path-loss gain of the Source-to-Relay $\left(S R_{i}\right)$ link and Relay-to-Destination $\left(R_{i} D\right)$ link with respect to the Source-toDestination $(S D)$ link are introduced here as $G_{s r_{i}}=\left\{\frac{D_{s d}}{D_{s r_{i}}}\right\}^{\alpha}$ and $G_{r_{i} d}=\left\{\frac{D_{s d}}{D_{r_{i} d}}\right\}^{\alpha}$, respectively, where $D_{a b}$ represents the distance between $a$ and $b$, while $\alpha$ is the path-loss exponent. More specifically, the value of $\alpha$ is fixed to 3 here to model a typical urban area. The transmit power of the node $a$ is represented as $P_{a}, a \in\left\{s, r_{0}, r_{1}\right\}$.

Then we assume that $N_{t}$ transmit antennas are used both at $s$, as well as at $r_{i}$, while $N_{r}$ receive antennas are employed at $d$. Due to the limited size and complexity of the handset, we fix $N_{t}=1$. Furthermore, the classic frame-by-frame based transmission having a frame length of $L$ routinely adopted in realistic communication networks is employed here.

The realistic transceiver architecture designed for each node involved in the SRAN is portrayed in Figure 2. For the sake of relating Figure 1 to Figure 2, the same transmissions are numbered by the same indices in the two figures. For example, since the transmission from the $\mathrm{SN}$ to the DN during the "(a) Even Phase" in Figure 1 corresponds to the transmission from the SN's transmit antenna to the receive antennae of the "Even Phase Multiple-Symbol DIS Filter" in Figure 2, both of them are labelled by the index 1 . Observe in Figure 1 that transmission-3 and transmission- 5 will contaminate the DN's signals received due to transmission- 1 and transmission- 4 , respectively. In order to mitigate this co-channel interference (CCI), the MS-DIS filter is employed at the input of the DN's receiver. The principle of the MS-DIS filter and the details conceiving other components involved in Figure 2 will be provided during our forthcoming discourse.

The system model corresponding to Figure 1 may be

\footnotetext{
${ }^{1}$ An arbitrary number of mobiles can be activated to act as relays. However, when more than two relays are assigned to the SRAN, the transmission arrangement is complicated. Hence, a classic SRAN with aid of two relays is employed in this paper for simplifying our analysis. More interpretation of the successive relaying protocol can be found in [8] and [14].
}

constructed for the $k^{t h}$ symbol duration of the $l^{\text {th }}$ frame as

$\mathbf{Y}^{l}[k]=\sqrt{G_{s d}} \mathbf{S}^{l}[k] \mathbf{H}_{s d}^{l}[k]+\sqrt{G_{r_{i} d}} \mathbf{C}_{r_{i}}^{l}[k] \mathbf{H}_{r_{i} d}^{l}[k]+\mathbf{W}^{l}[k]$,

where we have $\mathbf{Y}^{l}[k] \in \mathbb{C}^{1 \times N_{r}}, \mathbf{S}^{l}[k] \in \mathbb{C}^{1 \times N_{t}}, \mathbf{C}_{i}^{l}[k] \in$ $\mathbb{C}^{1 \times N_{t}}, \mathbf{H}_{a b}^{l}[k] \in \mathbb{C}^{N_{t} \times N_{r}}$, while $\mathbf{W}^{l}[k] \in \mathbb{C}^{1 \times N_{r}}$ denotes the $k^{t h}$ received signal matrix at the DN, the SN's broadcast symbol matrix, the RN's forwarded symbol matrix and the associated CIR matrix as well as the AWGN matrix obeying a distribution of $\mathcal{C N}\left(0,2 \delta_{w}^{2}\right)$, respectively. The notation $i$ is calculated as $i=\bmod [(l+1), 2]$ and $l=0,1, \cdots$.

In order to invoke the MS-DIS regime of [12] and to further combine it with the SISO-MSDSD algorithm [3], it is necessary to extend the symbol-by-symbol based system model characterized by (1) to its multiple-symbol based version. We split the DN's $L$ received signals of a frame into $J$ signal blocks, where each signal block consists of $N_{\text {wind }}$ consecutively received $\mathbf{Y}^{l}[k]$ contributions. Accordingly, the $n^{t h}$ signal block matrix received during the $l^{t h}$ frame may be readily formulated as

$$
\underline{\mathbf{Y}}^{l}[n]=\sqrt{G_{s d}} \underline{\mathbf{S}}^{l}[n] \underline{\mathbf{H}}_{s d}^{l}[n]+\sqrt{G_{r_{i} d}} \underline{\mathbf{C}}_{r_{i}}^{l}[n] \underline{\mathbf{H}}_{r_{i} d}^{l}[n]+\underline{\mathbf{W}}^{l}[n],
$$

where $\underline{\mathbf{Y}}^{l}[n]$ obeys the form of

$$
\underline{\mathbf{Y}}^{l}[n]=\left[\begin{array}{c}
\mathbf{Y}^{l}\left[L \cdot l+\left(N_{\text {wind }}-1\right) \cdot n\right] \\
\vdots \\
\mathbf{Y}^{l}\left[L \cdot l+\left(N_{\text {wind }}-1\right) \cdot(n+1)\right]
\end{array}\right] .
$$

As revealed by (3), the last element of $\underline{\mathbf{Y}}^{l}[n]$ will become the first element of $\underline{\mathbf{Y}}^{l}[n+1]$. This overlap between the two adjacent signal blocks is required by the MSDSD algorithm [3]. Then the CIR block $\underline{H}_{a b}^{l}[n]$ as well as the AWGN block $\underline{W}^{l}[n]$ are constructed by vertically stacking the associated $\overline{\mathbf{H}}_{a b}^{l}[k]$ and $\mathbf{W}^{l}[k]$ in a form similar to (3), respectively. Furthermore, the $n^{\text {th }}$ SN broadcast symbol block is given by $\underline{\mathbf{S}}^{l}[n]=$ $\operatorname{diag}\left\{\left[\mathbf{S}^{l}\left[L \cdot l+\left(N_{\text {wind }}-1\right) \cdot n\right]^{T}, \cdots, \mathbf{S}^{l}\left[L \cdot l+\left(N_{\text {wind }}-\right.\right.\right.\right.$ 1) $\left.\left.\cdot(n+1)]^{T}\right]^{T}\right\}$. An $\mathcal{M}$-ary differential encoding scheme is employed here, hence we have $\mathbf{S}^{l}[k+1]=\mathbf{S}^{l}[k] \mathbf{V}^{l}[k]$ and $\underline{\mathbf{S}}^{l}[n]$ will be uniquely determined by the length- $\left(N_{\text {wind }}-1\right)$ information symbol block matrix $\underline{\mathbf{V}}^{l}[n]=\left[\mathbf{V}^{l}\left[L \cdot l+\left(N_{\text {wind }}-\right.\right.\right.$ 1) $\left.\cdot n]^{T}, \cdots, \mathbf{V}^{l}\left[L \cdot l+\left(N_{\text {wind }}-1\right) \cdot(n+1)-1\right]^{T}\right]^{T}$.

The decode-and-forward (DF) relaying protocol is employed at the RN. If the RN perfectly detects the received information bits and re-encodes, as well as re-modulates them in the same way as the $\mathrm{SN}$, we arrive at

$$
\underline{\mathbf{C}}_{r_{i}}^{l}[n]=\underline{\mathbf{S}}^{l-1}[n] .
$$

\section{Adaptive Multiple-Symbol Based Differential INTERFERENCE SUPPRESSION}

According to (2), it is apparent that the transmitted signals of the SN and RN always interfere with each other at the DN. Hence noncoherent detection cannot be directly applied at the DN, before an interference suppression stage is invoked at the input of the DN's receiver. When we want to detect the signal received from the SN, the first term of (2) becomes the desired signal component, while the remaining terms represent 
the interference-plus-noise component. This partitioning of the received signal block matrix $\underline{\mathbf{Y}}^{l}[n]$ is detailed as

$$
\underline{\mathbf{Y}}^{l}[n]=\underbrace{\sqrt{G_{s d}} \underline{\mathbf{S}}^{l}[n] \underline{\mathbf{H}}_{s d}^{l}[n]}_{\text {desired component: } \underline{\underline{Y}}_{g}^{l}[n]}+\underbrace{\sqrt{G_{r_{i} d}} \underline{\mathbf{C}}_{r_{i}}^{l}[n] \underline{\mathbf{H}}_{r_{i} d}^{l}[n]+\underline{\mathbf{W}}^{l}[n]}_{\text {interference-plus-noise component }}
$$

Hence our goal is to extract the information pertaining to the desired signal component $\underline{\mathbf{Y}}_{g}^{l}[n]$ from the complete received signal block matrix $\underline{\mathbf{Y}}^{l}[n]$, while suppressing the interferenceplus-noise component. The adaptive MS-DIS technique of [12] is capable of realizing our objective and consequently it is invoked at the input of the DN's receiver, as we will detail in Section IV with reference to Figure 2.

\section{A. Maximum Signal-to-Interference-Plus-Noise Ratio Crite- rion}

In order to extract the $N_{\text {wind }}$ differentially encoded symbols pertaining to $\underline{\mathbf{Y}}_{g}^{l}[n]$, the received signal block matrix $\underline{\mathbf{Y}}^{l}[n]$ is passed through an adaptive filter having the coefficient vector $\boldsymbol{f}_{g}$ of length $N_{r}$. The filter output is given by

$$
\mathbf{y}_{g}^{l}[n]=\underline{\mathbf{Y}}^{l}[n] \boldsymbol{f}_{g} .
$$

According to [11], the signal-to-interference-plus-noise-ratio (SINR) is defined as the ratio between the power of the desired filter output component $\underline{\mathbf{Y}}_{g}^{l}[n] \boldsymbol{f}_{g}$ and the power of the interference-plus-noise component $\left(\underline{\mathbf{Y}}^{l}[n]-\underline{\mathbf{Y}}_{g}^{l}[n]\right) \boldsymbol{f}_{g}$. Hence, based on the maximum SINR (MSINR) criterion, our goal becomes that of finding a coefficient vector $\boldsymbol{f}_{g}$, which is capable of maximising the filter's output SINR. The decision rule of the optimum $\boldsymbol{f}_{g}$ is formulated as

$$
\boldsymbol{f}_{g}=\max _{\hat{\boldsymbol{f}}_{g}} \frac{\boldsymbol{f}_{g}^{H}\left(\mathbf{R}_{r r}-\mathbf{R}_{e e}\right) \boldsymbol{f}_{g}}{\boldsymbol{f}_{g}^{H} \mathbf{R}_{e e} \boldsymbol{f}_{g}}=\max _{\hat{\boldsymbol{f}}_{g}} \frac{\boldsymbol{f}_{g}^{H} \mathbf{R}_{r r} \boldsymbol{f}_{g}}{\boldsymbol{f}_{g}^{H} \mathbf{R}_{e e} \boldsymbol{f}_{g}},
$$

where the correlation matrix of the entire received signal block and of the interference-plus-noise component are defined by

$$
\begin{aligned}
\mathbf{R}_{r r} & \triangleq \mathcal{E}\left\{\underline{\mathbf{Y}}^{l^{H}}[n] \underline{\mathbf{Y}}^{l}[n]\right\} \\
& =\sum_{a b \in s d, r_{i} d} \underline{\mathbf{H}}_{a b}^{l}{ }^{H}[n] \underline{\mathbf{H}}_{a b}^{l}[n]+N_{t} N_{w i n d} 2 \delta_{w}^{2} \mathbf{I}_{N_{r}}, \\
\mathbf{R}_{e e} & \triangleq \mathcal{E}\left\{\left(\underline{\mathbf{Y}}^{l}[n]-\underline{\mathbf{Y}}_{g}^{l}[n]\right)^{H}\left(\underline{\mathbf{Y}}^{l}[n]-\underline{\mathbf{Y}}_{g}^{l}[n]\right)\right\} \\
& =\underline{\mathbf{H}}_{r_{i} d}^{l}{ }^{H}[n] \underline{\mathbf{H}}_{r_{i} d}^{l}[n]+N_{t} N_{w i n d} 2 \delta_{w}^{2} \mathbf{I}_{N_{r}},
\end{aligned}
$$

respectively. Then $\mathbf{I}_{N_{r}}$ represents an identity matrix having $\left(N_{r} \times N_{r}\right)$ elements. Furthermore, $\mathcal{E}\{\cdot\}$ and $[\cdot]^{H}$ denote the statistical expectation and the Hermitian transposition, respectively.

It was shown in [16] that the optimization problem of (7) leads to the generalized eigenvalue problem, which is formulated as

$$
\mathbf{R}_{r r} \boldsymbol{f}_{g}=\lambda \mathbf{R}_{e e} \boldsymbol{f}_{g}
$$

\section{B. Adaptive Modified Newton Algorithm}

According to (8) and (9), the CIR block matrix $\underline{\mathbf{H}}_{a b}^{l}[n]$ has to be known by the receiver for the sake of calculating the correlation matrices $\mathbf{R}_{r r}$ and $\mathbf{R}_{e e}$, which inherently leads to the employment of coherent detection. Then, even through the receiver already acquired the CIR block matrix $\underline{\mathbf{H}}_{a b}^{l}[n]$, we have to utilize the singular-value decomposition (SVD) [17] to implement the generalized eigen-decomposition for finding the optimum $\boldsymbol{f}_{g}$, which usually imposes a high computational complexity.

Alternatively, instead of obtaining the precise correlation matrices $\mathbf{R}_{r r}$ and $\mathbf{R}_{e e}$, we can recursively approximate them and update the sub-optimum coefficient vector $\boldsymbol{f}_{g}[n]$ on a block-by-block basis according to the adaptive modified Newton algorithm, which was shown in [13] to have a fast convergence and an excellent tracking capability.

Similar to $[11,(11)]$, by exploiting the differential encoding principle, the multiple-symbol-based error signal block matrix $\underline{\mathbf{E}}_{g}^{l}[n]$ may be expressed as

$$
\underline{\mathbf{E}}_{g}^{l}[n]=\sqrt{\frac{1}{2}}\left(\underline{\mathbf{Y}}^{l}[n]-\underline{\hat{\mathbf{V}}}_{g}^{l}[n] \cdot \underline{\mathbf{Y}}^{l}\left[\overleftarrow{n}^{(1)}\right]\right)
$$

which may be used to approximate the interference-plus-noise component of (5). The operation "." denotes the submatrixwise multiplication between two matrices. Then the specific block index $\overleftarrow{n}^{(m)}$ represents the $n^{\text {th }}$ signal block, which was shifted backwards by $m$ symbol durations. Furthermore, $\hat{\mathbf{V}}_{g}^{l}[n]=\left[\mathbf{V}^{l}\left[L \cdot l+\left(N_{\text {wind }}-1\right) \cdot n-1\right]^{T}, \underline{\mathbf{V}}^{l}[n]^{T}\right]^{T}$ is the $n^{\text {th }}$ transmitted information symbol block corresponding to the desired component of (5), which becomes known to the receiver in the training model of the adaptive filter or is actually substituted by the previous decisions in the decisiondirected model of the adaptive filter. The normalization factor $\sqrt{1 / 2}$ is compensated according to [11].

Based on (11), the correlation matrices $\mathbf{R}_{r r}$ and $\mathbf{R}_{e e}$ may be recursively estimated for the $n^{\text {th }}$ length - $N_{\text {wind }}$ signal block in the same way as in [13, (28-29)] without any knowledge of the CSI as follows

$$
\begin{aligned}
& \mathbf{R}_{r r}[n]=\beta \mathbf{R}_{r r}[n-1]+(1-\beta) \underline{\mathbf{Y}}^{l^{H}}[n] \underline{\mathbf{Y}}^{l}[n], \\
& \mathbf{R}_{e e}[n]=\mu \mathbf{R}_{e e}[n-1]+(1-\mu) \underline{\mathbf{E}}_{g}^{l}{ }^{H}[n] \underline{\mathbf{E}}_{g}^{l}[n],
\end{aligned}
$$

where $0<\beta, \mu<1$ are the forgetting factors.

Based on (12) and (13), the adaptive modified Newton algorithm is derived in [13]. For reasons of space economy we simply summarize the major steps as follows [13]

$$
\begin{aligned}
\mathbf{P}[n] & =\frac{1}{\mu} \mathbf{P}[n-1]\left(\mathbf{I}_{N_{r}}-\frac{\underline{\mathbf{E}}_{g}^{l H}[n] \underline{\mathbf{E}}_{g}^{l}[n] \mathbf{P}[n-1]}{\operatorname{tr}\left(\mu \mathbf{I}+\underline{\mathbf{E}}_{g}^{l}[n] \mathbf{P}[n-1] \underline{\mathbf{E}}_{g}^{l H}[n]\right)}\right) \\
\mathbf{c}[n] & =\underline{\mathbf{Y}}^{l}[n] \boldsymbol{f}_{g}[n-1], \\
\mathbf{r}[n] & =\beta \mathbf{r}[n-1]+(1-\beta) \underline{\mathbf{Y}}^{l^{H}}[n] \mathbf{c}[n], \\
d[n] & =\beta d[n-1]+(1-\beta) \mathbf{c}^{H}[n] \mathbf{c}[n], \\
\tilde{\boldsymbol{f}}_{g}[n] & =\frac{\mathbf{r}[n]}{d[n]}, \\
\boldsymbol{f}_{g}[n] & =\frac{2 \mathbf{P}[n] \tilde{\boldsymbol{f}}_{g}[n]}{1+\tilde{\boldsymbol{f}}_{g}^{H}[n] \mathbf{P}[n] \tilde{\boldsymbol{f}}_{g}[n]},
\end{aligned}
$$

where the initialization of the relevant parameters is implemented as: set $\mathbf{P}[0]=\mu_{1} \mathbf{I}_{N_{r}}, \boldsymbol{f}_{g}[0]=\mathbf{r}[0]=\mu_{2}[1,1, \cdots, 1]^{T}$ and $d[0]=\mu_{3}$, where $\mu_{i}(i=1,2,3)$ are appropriate positive values. The appropriate adaptive filter coefficient vector $\boldsymbol{f}_{g}[n]$ associated with the desired component of the $n^{\text {th }}$ received 
signal block matrix can be acquired by recursively implementing the procedure of (14). Consequently, the suppression of the interference-plus-noise component dispensing with CSI becomes possible.

\section{Relay-Aided SISO-MSDSD}

The SISO-MSDSD algorithm advocated by Pauli et al. in [3] is invoked for the noncoherent detection based iterative decoding scheme in the direct transmission scenario. However, the application of the SISO-MSDSD algorithm in multiple access systems had not been considered in their original work. The further development of the SISO-MSDSD algorithm for simultaneously detecting multiple input signal streams was outlined in [18], where the multiple input signal streams were expected to correspond to the same modulated symbols. This condition is usually satisfied in the specific family of cooperative networks, where the same channel coding and modulation schemes are adopted both at the SN and at the RNs. Hence a modified SISO-MSDSD algorithm has to be conceived for the cooperative network, which we refer to as Relay-Aided SISOMSDSD. The proposed Relay-Aided SISO-MSDSD algorithm evaluates the a posteriori log-likelihood Ratios (LLR) of the $\eta^{t h}$ bit $u[\eta]$ conditioned on $\Omega$ received signal streams represented by $\left\{\mathbf{y}_{\lambda}\right\}_{\lambda=1,2, \cdots, \Omega}$ as follows

$$
L_{u}[\eta]=\ln \frac{\operatorname{Pr}\left(u[\eta]=b \mid\left\{\mathbf{y}_{\lambda}\right\}_{\lambda=1,2, \ldots, \Omega}\right)}{\operatorname{Pr}\left(u[\eta]=\bar{b} \mid\left\{\mathbf{y}_{\lambda}\right\}_{\lambda=1,2, \ldots, \Omega}\right)}, \quad b \in\{0,1\},
$$

where $\bar{b}$ is the complement of the bit $b$.

The adaptive filter coefficient vector $\boldsymbol{f}_{g}[n]$ conditioned for extracting the differentially encoded symbols pertaining to the SN broadcast signals is specifically referred to as $\boldsymbol{f}_{s}[n]$. Correspondingly, $\boldsymbol{f}_{g}[n]$ adjusted for extracting the differentially encoded symbols pertaining to the RN's forwarded signals is denoted by $\boldsymbol{f}_{r_{i}}[n]$. Assuming the validity of (4) and substituting (4) into (2), the two received signal blocks having the same block index $n$ but two different frame indices of $l$ and $(l+1)$ can be further formulated as

$$
\begin{aligned}
\underline{\mathbf{Y}}^{l}[n] & =\sqrt{G_{s d}} \underline{\mathbf{S}}^{l}[n] \underline{\mathbf{H}}_{s d}^{l}[n]+\underline{\mathbf{I}}^{l}[n]+\underline{\mathbf{W}}^{l}[n], \\
\underline{\mathbf{Y}}^{l+1}[n] & =\sqrt{G_{r_{i}} d} \underline{\mathbf{S}}^{l}[n] \underline{\mathbf{H}}_{r_{i} d}^{l+1}[n]+\underline{\mathbf{I}}^{l+1}[n]+\underline{\mathbf{W}}^{l+1}[n],
\end{aligned}
$$

where $\underline{\mathbf{I}}^{l}[n]$ represents the associated interference component.

After $\underline{\mathbf{Y}}^{l}[n]$ and $\underline{\mathbf{Y}}^{l+1}[n]$ are passed through the adaptive filter and multiplied by $\boldsymbol{f}_{s}[n]$ and $\boldsymbol{f}_{r_{i}}[n]$, respectively, the filter outputs are given by

$$
\begin{aligned}
\mathbf{y}_{s}^{l}[n] & =\underline{\mathbf{Y}}^{l}[n] \boldsymbol{f}_{s}[n] \\
\mathbf{y}_{r_{i}}^{l+1}[n] & =\underline{\mathbf{Y}}^{l+1}[n] \boldsymbol{f}_{r_{i}}[n] .
\end{aligned}
$$

Both $\mathbf{y}_{s}^{l}[n]$ and $\mathbf{y}_{r_{i}}^{l+1}[n]$ correspond to the differentially encoded symbol block $\underline{\mathbf{S}}^{l}[n]$. Hence we substitute them into (15) as $\left\{\mathbf{y}_{\lambda}\right\}_{\lambda=1,2}$, and (15) may be rewritten with the aid of Bayes' theorem as (18), where $\chi_{: u[\eta]=b}$ denotes the set of $\mathcal{M}^{\left(N_{\text {wind }}-1\right)} / 2$ legitimate transmitted symbol blocks $\underline{\mathbf{V}}^{l}[n]$, whose $\eta^{t h}$ bit is constrained to $u[\eta]=b$. Similarly, $\chi_{: u[\eta]=\bar{b}}$ is defined as the set corresponding to $u[\eta]=\bar{b}$.

The optimum hypothesis of $\underline{\mathbf{V}}^{l}[n]$ can be attained by the ML detector and the corresponding performance is approached by the sphere detection algorithm, which significantly reduces the detection complexity and completes the calculation of $L_{u}[\eta]$ in (18). In order to avoid any repetition, for the further derivations and interpretations of the Relay-Aided SISO-MSDSD algorithm we refer to [18].

\section{TRansceiver Design and Analysis}

An appropriate practical transceiver is required now to implement the state-of-the-art techniques introduced in Section III, which efficiently organizes the collaboration amongst all functional blocks.

\section{A. Architecture Design and Analysis}

We specifically design a transceiver for the proposed DF aided SRAN, whose architecture is portrayed at Figure 2. In order to significantly enhance the error correction capability of the decoder, channel coding is invoked as an essential part of contemporary communication systems. The classic Convolutional Code (CC) and Recursive Systematic Convolutional (RSC) code constitute some of the most frequently employed schemes [3], [12]. Moreover, since it was demonstrated in [19] that Self-Concatenated Convolutional Codes (SECCC) [20] are capable of outperforming state-of-the-art benchmarks, a SECCC encoder is employed as the outer code at the SN. Then, the SECCC is combined with a differentially encoded modulator, which is further amalgamated with a unity-ratecode (URC) in order to create a two-stage inner code. Consequently, a three-stage SECCC-URC-DM source encoder was conceived.

Since it is unrealistic to expect that in addition to relaying, the RN could also estimate the channel $S R_{i}$, employing noncoherent detection dispensing with channel estimation at the RN seems to be more practical. Then the transmissions spanning the $S R_{i}$ link are similar to direct transmissions. Hence the single-path SISO-MSDSD scheme of [3] may be adopted as the first component decoder of the RN's receiver. Then the SISO-MSDSD soft decoder is further amalgamated with the URC decoder to create a two-stage MSDSD-URC inner decoder. In line with the SN's transmitter, the SECCC decoder is employed as the outer decoder of the RN's receiver. Consequently, a three-stage concatenated iterative decoder is used as the RN's receiver, as seen in the "Relay" block of Figure 2. Our motivation with the employment of this three-stage - rather than classic two-stage - concatenated architecture is to improve the attainable convergence behaviour of the iterative decoder with the aid of the URC decoder, as first proposed in [21] and detailed in [22]. Since the URC model has an infinite impulse response (IIR) as a benefit of its recursive encoder structure, it is capable of meritoriously reshaping the EXIT curve of the two-stage MSDSD-URC inner decoder to approach the point of perfect convergence at $(1,1)$ in the EXIT chart, which implies that an infinitesimally low error probability is attained. Consequently, a potentially critical impediment of the DF protocol - namely its error propagation may be avoided and (4) is satisfied. The transmitter of the RN may be designed to obey exactly the same architecture as that of the SN. Furthermore, the notations $E(\cdot)$ and $A(\cdot)$ denote the extrinsic information and a priori information, respectively, which are iteratively interleaved and exchanged $I_{\text {inner }}^{r}$ times 


$$
L_{u}[\eta]=\ln \frac{\operatorname{Pr}\left(u[\eta]=b \mid \mathbf{y}_{s}^{l}[n], \mathbf{y}_{r_{i}}^{l+1}[n]\right)}{\operatorname{Pr}\left(u[\eta]=\bar{b} \mid \mathbf{y}_{s}^{l}[n], \mathbf{y}_{r_{i}}^{l+1}[n]\right)}=\ln \frac{\sum_{\underline{\mathbf{V}}^{l}[n] \in \chi_{: u[\eta]=b}} \operatorname{Pr}\left(\mathbf{y}_{s}^{l}[n] \mid \underline{\mathbf{V}}^{l}[n]\right) \operatorname{Pr}\left(\mathbf{y}_{r_{i}}^{l+1}[n] \mid \underline{\mathbf{V}}^{l}[n]\right) \operatorname{Pr}\left(\underline{\mathbf{V}}^{l}[n]\right)}{\sum_{\underline{\mathbf{V}}^{l}[n] \in \chi_{: u[\eta]=\bar{b}}} \operatorname{Pr}\left(\mathbf{y}_{s}^{l}[n] \mid \underline{\mathbf{V}}^{l}[n]\right) \operatorname{Pr}\left(\mathbf{y}_{r_{i}}^{l+1}[n] \mid \underline{\mathbf{V}}^{l}[n]\right) \operatorname{Pr}\left(\underline{\mathbf{V}}^{l}[n]\right)}
$$

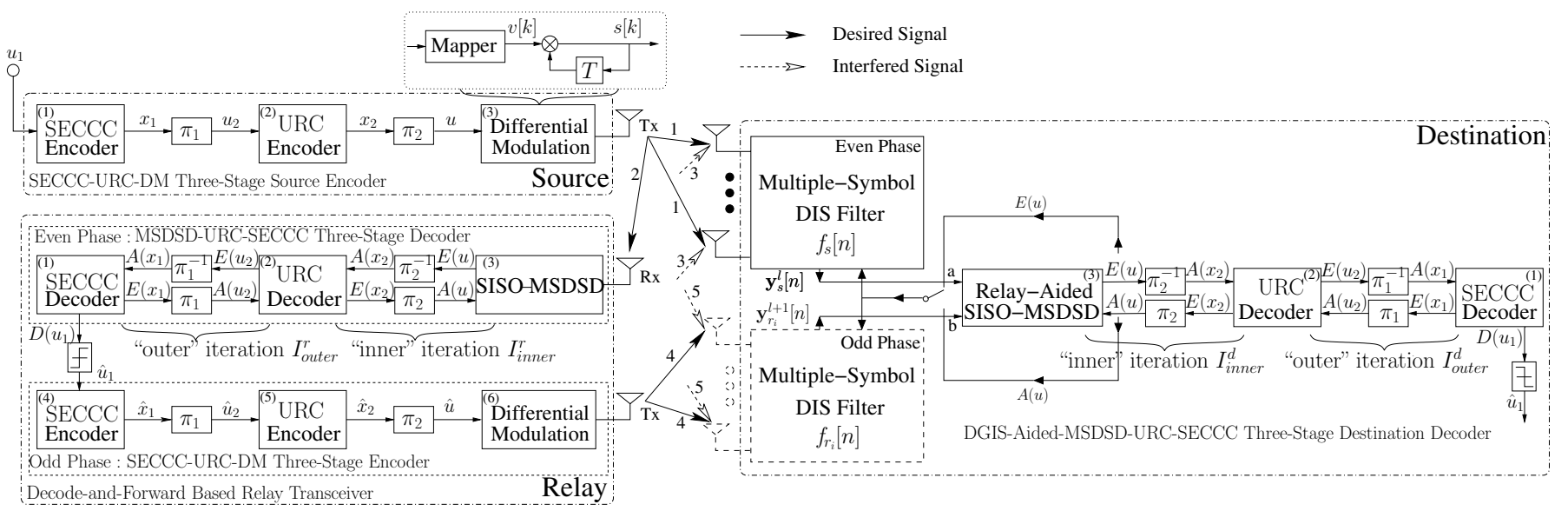

Fig. 2: The architecture of our proposed MS-DIS plus MSDSD assisted SECCC aided three-stage turbo transceiver

within the MSDSD-URC inner code and then passed on to the SECCC outer code. The resultant signal will be further iteratively interleaved and exchanged $I_{\text {outer }}^{r}$ times between the MSDSD-URC inner code and the SECCC outer code.

At the DN, we have to consider the interference problem as introduced in Section III-B. Then, we always obtain two replicas of the same SN's codeword, as analysed in Section III-C. Hence, in contrast to the RN's receiver, the MS-DIS filter is employed at the DN to carry out adaptive interference suppression. Meanwhile, instead of using a single-path SISOMSDSD decoder, the more sophisticated Relay-Aided SISOMSDSD decoder is employed at the DN to carry out the joint detection of the two input signal streams corresponding to the same codeword. To elaborate a little further, we observe the "Destination" block in Figure 2, while still focusing on the detection of the information pertaining to the SN's broadcast signals during the even-indexed transmission phase ( transmission-1 of Figure 2). Then the even-phase MS-DIS filter should aim for suppressing the interference imposed by the RN's forwarded signal component (labelled by transmission3 in Figure 2). This is realized by adaptively updating the filter coefficient vector $\boldsymbol{f}_{s}[n]$ of (17) according to the adaptive modified Newton algorithm detailed in (14) of Section III-B. Consequently, one of the expected filter outputs $\mathbf{y}_{s}^{l}[n]$ of (17) is generated. This even-phase MS-DIS filter should be activated during the even-phase. Then, another replica of the differentially encoded symbol block $\underline{\mathbf{S}}^{l}[n]$ of (5) pertains to the RN's forwarded signal block having the block index $n$ and frame index $(l+1)$ (transmission-4 of Figure 2). Hence, the odd-phase MS-DIS filter will be activated in the consecutive odd-phase to generate $\mathbf{y}_{r_{i}}^{l+1}[n]$ of Eq.(17) by updating the filter coefficient vector $\boldsymbol{f}_{r_{i}}[n]{ }^{2}$. Then, we concatenate the

\footnotetext{
${ }^{2}$ Actually, only one antenna array and one MS-DIS filter are activated at the beginning of the DN's action. The dual antenna arrays shown in Figure 2 and a pair of MS-DIS filters are used to emphasize the distinctive nature of the even and odd phases. Hence the antenna array and the MS-DIS filter corresponding to the odd phase are represented by dashed lines.
}

MS-DIS filter with the Relay-Aided SISO-MSDSD decoder and substitute $\mathbf{y}_{s}^{l}[n]$ and $\mathbf{y}_{r_{i}}^{l+1}[n]$ into (15) as $\left\{\mathbf{y}_{\lambda}\right\}_{\lambda=1,2}$. As seen in Figure 2, both the soft output of the Relay-Aided SISO-MSDSD decoder at the DN and that of the SISOMSDSD decoder at the RN correspond to the same coded bit stream " $u$ ". Hence the remaining architectural features of our DN's receiver seen in Figure 2 are arranged in the same way according to the corresponding part of the RN's receiver.

\section{B. Turbo Equalization Based Decoding Regime}

The turbo equalization philosophy originally proposed in [23] and detailed in [24] combines the equalization technique with the iterative detection process, where the equalization process is activated during each and every iteration of the turbo receiver. As a benefit of the gradually increased reliability of the feedback decisions, the performance of the equalizer may be significantly improved upon increasing the number of iterations. According to the turbo equalization principle, the MS-DIS filter will process the same received signal frame again with the aid of the most recent feedback decisions in each and every inner iteration of the two-stage MSDSD-URC inner code. As proposed in [12], the soft-symbol-decisiondirected feedback philosophy is adopted and the coefficient vector $\boldsymbol{f}_{g}[n]$ is updated on a block-by-block basis to reduce the complexity imposed. For the sake of training the adaptive equalizer, a training sequence constituted by legitimate differentially encoded symbols known by the DN is periodically transmitted by the $\mathrm{SN}$ according to a certain training overhead. Hence, in the training mode, the MS-DIS filter exploits the knowledge of the training symbols to adapt its coefficient vector. Naturally, we may argue that these training symbols can also be utilized for estimating the CSI and consequently coherent detection becomes feasible. Nevertheless, it will be demonstrated in Section VI that the training overhead required by our proposed system is low, which would fail to facilitate the Nyquist-sampling based channel estimation of the channels considered at realistic Doppler frequencies. Then, in the 
decision-directed mode of the MS-DIS filter, both the extrinsic information provided by the relay-aided MSDSD decoder and the a priori information provided by the URC decoder may be utilized as the soft decision feedback for updating the coefficient vector. Hence, a supplemental information quantity comparison between the extrinsic information and the a priori information is needed, so that the higher of the two may be exploited.

In more detail, during the first inner iteration, i.e for $I_{\text {inner }}^{d}=1, I_{\text {outer }}^{d}=1$, the MS-DIS filter first invokes the current coefficient vector for suppressing the interference and then updates its coefficient vector based on the current feedback decisions. This implies that the coefficient vector generated during the $n^{\text {th }}$ block is actually utilized during the $(n+1)^{t h}$ block. Furthermore, in this iteration, the switch shown in Figure 2 should be set to point "a", since the $a$ priori information provided by the URC decoder equals to zero.

During the consecutive iterations, the MS-DIS filter will first update its coefficient vector based on the feedback decisions generated during the most recent iteration and then utilizes the updated coefficient vector to suppress the interference. This implies that the coefficient vector generated in the $n^{\text {th }}$ block is immediately exploited within the same block. Then, the switch will oscillate between the points "a" and "b".

\section{NONCOHERENT DCMC CAPACITY FOR THE DF BASED SRAN IN MULTIPLE-ANTENNA SCENARIO}

In this contribution, we conceive an interference suppression aided DF based SRAN, where a MSDSD based noncoherent detection scheme is employed. Hence the noncoherent DCMC capacity of the DF based SRAN can be utilized as a benchmark to assess the performance of the transceiver portrayed in Figure 2 . The capacity derivation process of this section hinges on the assumptions related to the DN's receiver employed in the forthcoming simulation part.

The transmission arrangement of the twin-relay-aided successive relaying procedure may be viewed as the superposition of the transmissions of two conventional single-relay aided two-phase cooperative networks[14]. This is also illustrated in Figure 1, where the transmissions represented by the solid lines in the "(a) Even Phase" and "(b) Odd Phase" (labelled by "1", "2", "4") constitute one of the conventional singlerelay aided two-phase cooperative networks, namely Coop-I. Similarly, the transmissions represented by the dashed lines in the "(b) Odd Phase" and "(c) Even Phase" constitute another one, namely Coop-II. According to this philosophy, we can readily derive the upper and lower bounds on the noncoherent DCMC capacity of our SRAN as

$$
\begin{aligned}
\left\{C_{\text {Coop-I }}^{\text {DF-Uper }}+C_{\text {Coop-II }}^{\text {DF-Upper }}\right\} & \geq C_{\text {Successive }}^{\text {DF }} \\
C_{\text {Successive }}^{\text {DF }} & \geq\left\{C_{\text {Coop-I }}^{\text {DF-Lower }}+C_{\text {Coop-II }}^{\text {DF-Lower }}\right\},
\end{aligned}
$$

where the notations $C_{\text {Coop-I }}^{\text {DF-Uper }}$ and $C_{\text {Coop-I }}^{\text {DF-Lower }}$ represent the upper and lower bounds on the noncoherent DCMC capacity of the sub-network "Coop-I", respectively. The capacities $C_{\text {Coop-II }}^{\text {DF-Upper }}$ and $C_{\text {Coop-II }}^{\text {DF-Lower }}$ are defined in a similar way.

Firstly, we concentrate on the sub-network Coop-I. Based on the analysis provided in [25] and on the fact that the $\mathrm{SN}$ remains silent during the cooperative phase, we can obtain the upper and lower bounds for the sub-network Coop-I as

$$
\begin{aligned}
& C_{\text {Coop-I }}^{\text {DF-Upper }}=\min \left\{\frac{\theta_{1}}{T_{b}} I\left(\underline{\mathbf{S}}^{l}[n] ; \underline{\mathbf{Y}}^{l}[n], \underline{\mathbf{z}}_{r_{0}}^{l}[n]\right),\right. \\
& \left.\frac{\theta_{1}}{T_{b}} I\left(\underline{\mathbf{S}}^{l}[n] ; \underline{\mathbf{Y}}^{l}[n]\right)+\frac{\theta_{2}}{T_{b}} I\left(\underline{\mathbf{C}}_{r_{0}}^{l+1}[n] ; \underline{\mathbf{Y}}^{l+1}[n]\right)\right\} ; \\
& C_{\text {Coop-I }}^{\text {DF-Lower }}=\min \left\{\frac{\theta_{1}}{T_{b}} I\left(\underline{\mathbf{S}}^{l}[n] ; \underline{\mathbf{Z}}_{r_{0}}^{l}[n]\right),\right. \\
& \left.\frac{\theta_{1}}{T_{b}} I\left(\underline{\mathbf{S}}^{l}[n] ; \underline{\mathbf{Y}}^{l}[n]\right)+\frac{\theta_{2}}{T_{b}} I\left(\underline{\mathbf{C}}_{r_{0}}^{l+1}[n] ; \underline{\mathbf{Y}}^{l+1}[n]\right)\right\},
\end{aligned}
$$

where $\underline{\mathbf{Z}}_{r_{0}}^{l}[n]$ represents the $n^{\text {th }}$ signal block received at the RN $r_{0}$ during the $l^{\text {th }}$ frame, which consists of $N_{\text {wind }}$ consecutively received signals of the RN. Moreover, $\underline{\mathbf{Y}}^{l}[n]$ and $\underline{\mathbf{Y}}^{l+1}[n]$ are defined in (16), and $\underline{\mathbf{C}}_{r_{0}}^{l+1}[n]$ is defined in (2), where $\theta_{1}$ and $\theta_{2}$ are introduced to represent the relative lengths of the broadcast duration and the cooperative duration of subnetwork Coop-I, respectively, when their sum is normalized to unity. In contrast to the single antenna aided conventional three-stage receiver of the RN, the DN's receiver has multiple antennas and the novel architecture shown in Figure 2, which now becomes our focus. In order to concentrate on the DN's characteristics, we stipulate the idealized simplifying assumption that the relays always achieve perfect detection ${ }^{3}$, which implies that

$$
\begin{aligned}
\theta_{1} I\left(\underline{\mathbf{S}}^{l}[n] ; \underline{\mathbf{Z}}_{r_{0}}^{l}[n]\right) \geq & \theta_{1} I\left(\underline{\mathbf{S}}^{l}[n] ; \underline{\mathbf{Y}}^{l}[n]\right)+ \\
& \theta_{2} I\left(\underline{\mathbf{C}}_{r_{0}}^{l+1}[n] ; \underline{\mathbf{Y}}^{l+1}[n]\right) .
\end{aligned}
$$

According to (4), and substituting (22) into (20), (21), we arrive at

$$
\begin{aligned}
C_{\text {Coop-I }}^{\text {DF-Upper }}=C_{\text {Coop-I }}^{\text {DF-Lower }}= & \frac{\theta_{1}}{T_{b}} I\left(\underline{\mathbf{S}}^{l}[n] ; \underline{\mathbf{Y}}^{l}[n]\right)+ \\
& \frac{\theta_{2}}{T_{b}} I\left(\underline{\mathbf{S}}^{l}[n] ; \underline{\mathbf{Y}}^{l+1}[n]\right),
\end{aligned}
$$

which implies that, owing to the assumption formulated in (22), the upper and lower bounds on the capacity of the sub-network Coop-I converge. According to the symmetry between the sub-networks Coop-I and Coop-II, it may be readily seen that the upper and lower bounds on the capacity of the sub-network Coop-II also converge. Hence, (19) eventually transforms to

$$
C_{\text {Successive }}^{\mathrm{DF}}=C_{\text {Coop-I }}^{\mathrm{DF}}+C_{\mathrm{Coop}-\mathrm{II}}^{\mathrm{DF}}=C_{\text {Coop-I }}^{\mathrm{DF}-\mathrm{L} \text {-Uer }}+C_{\text {Coop-II }}^{\mathrm{DF}-\text { Lower }} .
$$

Then, according to [26], the probability density function of $\underline{\mathbf{Y}}^{l}[n]$ conditioned on $\underline{\mathbf{S}}^{l}[n]$ is expressed as ${ }^{4}$

$$
\operatorname{Pr}(\underline{\mathbf{Y}} \mid \underline{\mathbf{S}})=\frac{\exp \left[-\operatorname{tr}\left(\underline{\mathbf{Y}}^{H} \mathbf{\Psi}^{-1} \underline{\mathbf{Y}}\right)\right]}{\left[\pi^{T_{b} N_{T}} \operatorname{det}(\mathbf{\Psi})\right]^{N_{R}}} .
$$

Based on the assumption that the interference components in (16) are perfectly eliminated with the aid of an adaptive

\footnotetext{
${ }^{3}$ Such idealized assumption will be adopted in the forthcoming simulation part for the sake of concentrating on the DN's characteristics. Hence, in order to relate the capacity analysis here to our simulation results, we have to maintain consistency between the theoretical and simulation part of the paper.

${ }^{4}$ In order to simplify the equations, the signal block index $n$ and the frame index $l$ were omitted from now on.
} 


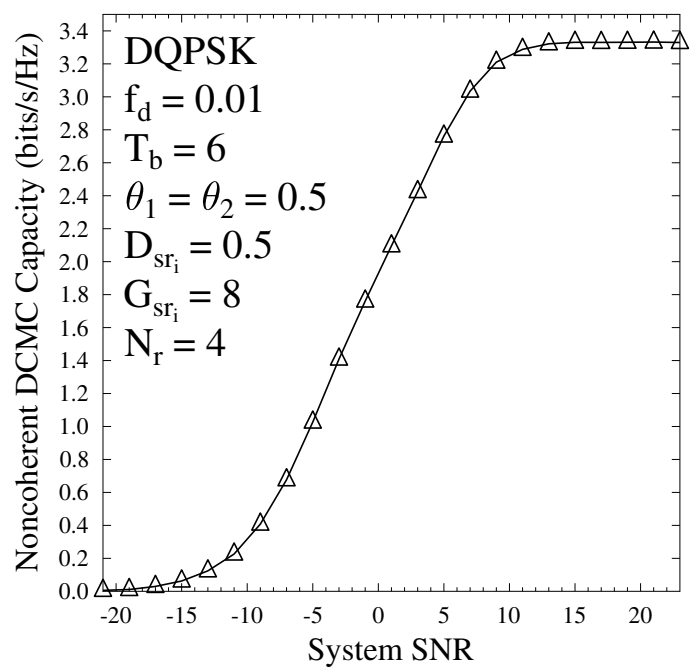

Fig. 3: The capacity of the cooperative phase in the DF based SRAN, where a normalized Doppler frequency of $f_{d}=0.01$ and a correlated block-fading period of $T_{b}=6$ are assumed.

interference cancellation technique, such as our MS-DIS, the conditional covariance matrix $\Psi$ may be calculated as

$$
\begin{aligned}
\boldsymbol{\Psi} & =\mathcal{E}\left\{\underline{\mathbf{Y}}^{H} \mid \underline{\mathbf{S}}\right\} \\
& =P_{s} G_{s d} \underline{\mathbf{S}} \mathcal{E}\left\{\underline{\mathbf{H}}_{s d} \underline{\mathbf{H}}_{s d}{ }^{H}\right\} \underline{\mathbf{S}}^{H}+N_{t} N_{r} 2 \sigma_{w}^{2} \mathbf{I}_{N_{w i n d}} .
\end{aligned}
$$

According to [25] and similar to [27], the first mutual information component on the right hand side of (23) can be attained through following process

$$
\begin{aligned}
I(\underline{\mathbf{S}} ; \underline{\mathbf{Y}}) & =H(\underline{\mathbf{Y}})-H(\underline{\mathbf{Y}} \mid \underline{\mathbf{S}}) ; \\
H(\underline{\mathbf{Y}} \mid \underline{\mathbf{S}}) & =\log _{2}\left\{\mathrm{e}^{T_{b}} \cdot[\operatorname{det}(\pi \mathbf{\Psi})]^{N_{R}}\right\} \\
H(\underline{\mathbf{Y}}) & =-\mathcal{E}\left\{\log _{2}\left[\sum_{\underline{\mathbf{S}}^{\prime} \in \chi} \frac{\exp \left[-\operatorname{tr}\left(\underline{\mathbf{Y}}^{H} \mathbf{\Psi}^{-1} \underline{\mathbf{Y}}\right)\right]}{M_{c}^{T_{b}}[\operatorname{det}(\pi \mathbf{\Psi})]^{N_{R}}}\right]\right\} .
\end{aligned}
$$

Similarly, the second mutual information component on the right hand side of (23) can be attained by implementing the above process, where the DN's received signal block $\underline{\mathbf{Y}}^{l+1}[n]$ is involved this time and $\Psi$ has to be modified to

$$
\boldsymbol{\Psi}=P_{r_{0}} G_{r_{0} d} \underline{\mathbf{S}} \mathcal{E}\left\{\underline{\mathbf{H}}_{r_{0} d} \underline{\mathbf{H}}_{r_{0} d}{ }^{H}\right\} \underline{\mathbf{S}}^{H}+N_{t} N_{r} 2 \sigma_{w}^{2} \mathbf{I}_{N_{w i n d}} .
$$

According to (23), (27), (26) and (28), the noncoherent DCMC capacity bounds $C_{\text {coop-I }}^{\mathrm{DF}-\mathrm{I} p e r}$ and $C_{\text {coop-I }}^{\mathrm{DF}-\mathrm{L} \text { erer }}$ of the subnetwork Coop-I can now be derived. Then, we can simply attain $C_{\text {coop-II }}^{\text {DF-Uper }}$ and $C_{\text {coop-II }}^{\text {DF-Low }}$ following a similar process. As a result, the noncoherent DCMC capacity $C_{\text {successive }}^{\mathrm{DF}}$ of the DF based SRAN given by (24) becomes available. Assuming that we have $D_{s r_{i}}=\frac{1}{2}$ and $\theta_{1}=\theta_{2}=\frac{1}{2}$, as well as that $N_{r}=4$ antennas are employed by the DN's receiver, the

\begin{tabular}{|c|c|}
\hline Channel Model & Time-Selective Rayleigh Fading Channel \\
\hline Path-Loss Exponent & $\alpha=3$ \\
\hline Normalized Doppler Frequency & $f_{d}=0.01$ \\
\hline Relay Position & $\overline{D_{s r_{i}}}=\frac{1}{2} ; G_{s r_{i}}=G_{r_{i} d}=8$ \\
\hline Channel Coding & SECCC \\
\hline Memory Length of SECCC & $\nu=6$ \\
\hline Puncture Rate of SECCC & $p=0.5$ \\
\hline Iteration Number of SECCC & $I_{S E C C C}=2$ \\
\hline Code Rate of SECCC & $R_{c}=0.5$ \\
\hline Interleaver Length & $L=144000$ bits \\
\hline Modulation & DQPSK \\
\hline MSDSD Observation Window Size & $N_{\text {wind }}=6$ \\
\hline Inner Iterations of DN's Decoder & $I_{\text {inner }}^{d}=2$ \\
\hline Outer Iterations of DN's Decoder & $I_{\text {outer }}^{d}=9$ \\
\hline Overall Bandwidth efficiency & $\begin{array}{r}\eta \approx R_{c} \cdot \log _{2} M_{c} \cdot \frac{T_{b}-1}{T_{b}} \\
=0.833 \mathrm{bits} / \mathrm{s} / \mathrm{Hz}\end{array}$ \\
\hline Optimum Forgetting Factor & $\beta=0.995 ; \mu=0.30$ \\
\hline Training Overhead & $2 \%$ \\
\hline Initialization of MS-DIS Filter & $\mu_{1}=0.01 ; \mu_{2}=\frac{1}{\sqrt{\left\|f_{g}[n]\right\|_{F}^{2}}} ; \mu_{3}=1$ \\
\hline Antenna Configuration & $N_{t}=1 ; N_{r}=4$ \\
\hline
\end{tabular}
associated $C_{\text {successive }}^{\mathrm{DF}}$ capacity is portrayed in Figure 3. In fact, the resultant capacity $C_{\text {successive }}^{\mathrm{DF}}$ as evaluated from (24), (23), (27), (26) and (28) is equivalent to the cooperative phase capacity of the DF based SRAN, where the potential 50\%
TABLE I: System Parameters

capacity loss of the DF based SRAN imposed by the section of the broadcast phase is eliminated owing to the assumption that the relays always achieve perfect detection, as formulated in (22).

\section{Vi. Simulation Results and Discussions}

Let us now report on our experiments, including the EXIT chart analysis of the SECCC outer code, the effects of the forgetting factors $(\beta, \mu)$, as well as the error correction capability of both the RN's and the BS's receiver. The major system parameters adopted in our simulations are summarized in Table $\mathrm{I}^{5}$.

\section{A. EXIT Chart Analysis of SECCC}

The EXIT curve of the SECCC outer code had been portrayed in [19], where the effects of diverse memory lengths $\nu$ and coding rates $R_{c}$ were characterized. The impact of the puncturing rate $p$ and of the number of inner iterations $I_{S E C C C}$ is characterized in Figure 4 as an extension of [19], where the EXIT curve of a classic RSC code having the same memory length and coding rate is provided as a benchmark.

Observe in Figure 4a that increasing the number of inner iterations between the two virtual component RSC decoders of the SECCC is capable of slightly improving the EXIT curve of the SECCC at the cost of imposing an increased complexity. Furthermore, it is shown in Figure $4 \mathrm{~b}$ that the optimum puncturing rate of the puncturer in the SECCC may be $1 / 2$ rather than $1 / 3,1 / 4$, since the former results in an improved EXIT performance. Based on these results we conclude that $I_{S E C C C}=2$ and $p=1 / 2$ constitute a competitive choice.

\section{B. Optimization of the Forgetting Factors}

The specific choice of the forgetting factors $(\beta, \mu)$ invoked in Section III-B significantly influences the performance of

\footnotetext{
${ }^{5}$ The system parameters involved in some particular experiments will be specifically interpreted, if they happen to be different from the default values provided in Table I.
} 


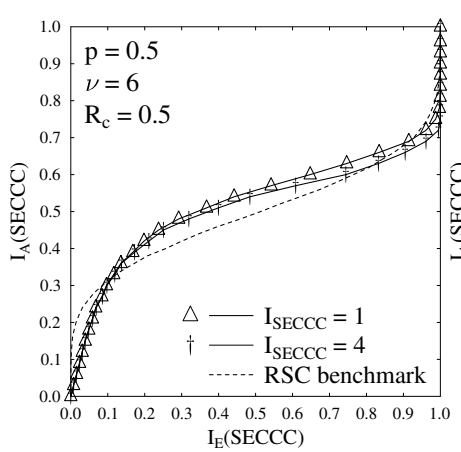

(a) Effects of the inner iterations

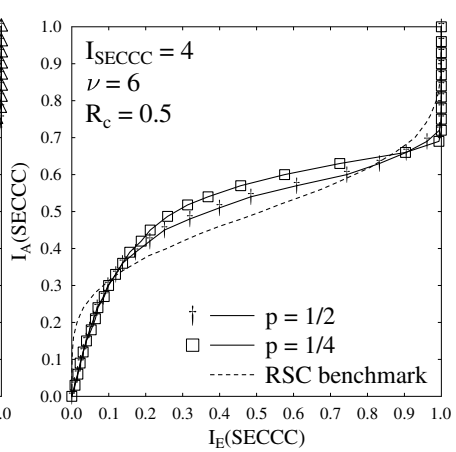

(b) Effects of the puncture rates

Fig. 4: The EXIT characteristic of the SECCC outer code for different number of inner iterations and puncturing rates.

the adaptive modified Newton algorithm, as mentioned in [13]. Hence finding the optimum forgetting factors associated with a specific scenario is required. It was shown in [28] that the area under the bit-based EXIT curve of the inner code approximates the maximum achievable coding rate of the outer channel code, while guaranteeing near-error-free communication. Hence we can utilize the EXIT curves of the MS-DIS aided MSDSD-URC inner codes having different forgetting factor values for the optimization of the forgetting factors. According to the above analysis, the EXIT curve assisted optimization of the forgetting factors is supported by the relevant results shown in Figure 5, where the DN relied on $N_{r}=4$ and a normalized Doppler frequency of $f_{d}=0.01$ was assumed.

The forgetting factors involved in the MS-DIS filters of the even and odd phases are represented by $\left(\beta_{0}, \mu_{0}\right)$ and $\left(\beta_{1}, \mu_{1}\right)$, respectively. Due to the particular transmission regime of the SRAN, if a differentially encoded symbol block $\underline{\mathbf{S}}^{l}[n]$ is broadcast by the $\mathrm{SN}$ in the even phase, then it will be forwarded by the RN in the consecutive odd phase. This implies that the roles of the signal components received by the $\mathrm{DN}$ from the $\mathrm{SN}$ and $\mathrm{RN}$ are reversed in the consecutive even and odd phases in terms of the desired component and the interference-plus-noise component. Hence, according to the functions of the forgetting factors $\beta$ and $\mu$ described by (12) and (13), it may be readily inferred that the values of $\left(\beta_{0}, \mu_{0}\right)$ and $\left(\beta_{1}, \mu_{1}\right)$ should also obey a reversed relationship, in order to realize a reasonable collaboration between the even-phase MS-DIS filter and odd-phase MS-DIS filter. This statement is verified by Figure $5 \mathrm{a}$, where the EXIT curves corresponding to the forgetting factors satisfying $\left(\beta_{1}, \mu_{1}\right)=\left(\beta_{0}, \mu_{0}\right)$ are shifted further down compared to the forgetting factors obeying $\left(\beta_{1}, \mu_{1}\right)=\left(\mu_{0}, \beta_{0}\right)$.

After confirming the optimization rule that $\left(\beta_{1}, \mu_{1}\right)$ should be equal to $\left(\mu_{0}, \beta_{0}\right)$, a deeper search conceived for finding the optimum forgetting factors with respect to our specific scenario may be invoked and the relevant results are displayed in Figure 5b. Observe in Figure 5b that the area under the EXIT curve of the MS-DIS aided MSDSD-URC inner code was consistently increased upon reducing the associated value of $\mu_{0}$. However, the improvement achieved by shifting the EXIT curve upwards upon reducing the value of $\mu_{0}$ became rather negligible for $\mu_{0}<0.30$. Based on
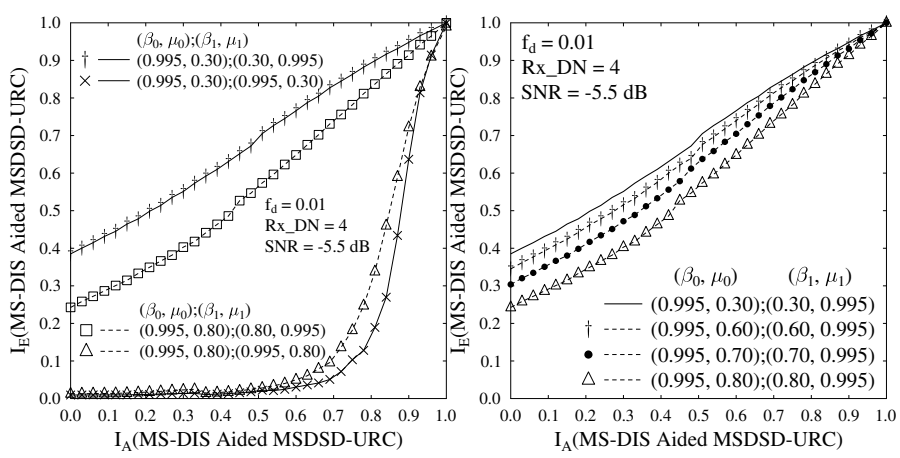

(a) Forgetting factor comparison-I

(b) Forgetting factor comparison-II

Fig. 5: EXIT-curve-assisted forgetting factor optimization.

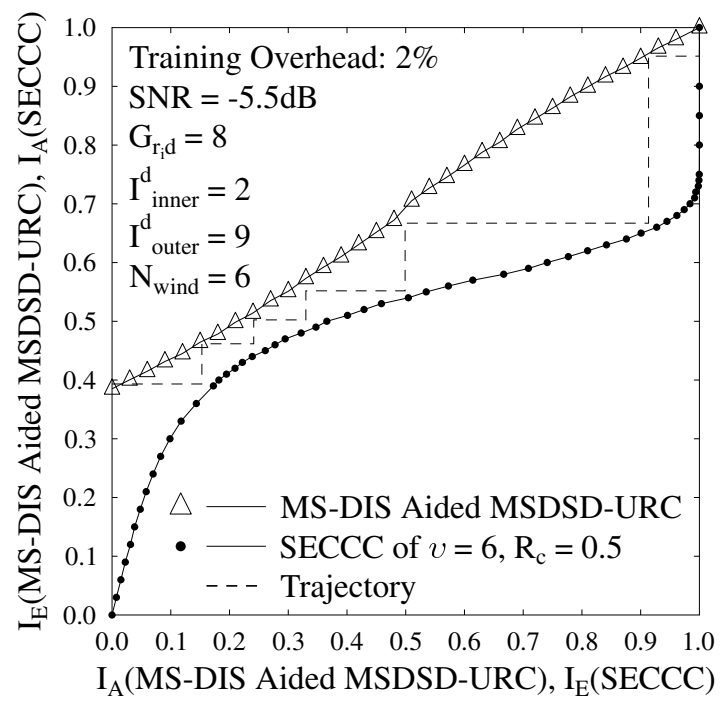

Fig. 6: The EXIT characteristics of our proposed BS receiver.

our detailed investigations not included here ${ }^{6}$, the forgetting factors $\left(\beta_{0}, \mu_{0}\right)=(0.995,0.30)$ were deemed as the best choice and were hence employed for the actual MS-DIS filter in our specific scenario.

\section{Error Correction Performance at the DN}

Again, the BS is capable of supporting a more sophisticated, but higher-complexity architecture than the relays. Accordingly, the number of BS receiver antennas is fixed to $N_{r}=4$ for enhancing its error correction capability with the aid of its increased spatial diversity gain. Then, in order to concentrate on the DN's characteristics, the idealized simplifying assumption invoked in Section V, namely that the relays always achieve perfect detection is adopted again. Consequently, our capacity analysis and simulation results provided in Section V can be utilized here ${ }^{7}$. Based on this assumption, the EXIT chart and the BER vs SNR performance of the proposed BS receiver are portrayed in Figure 6 and Figure 7, respectively.

\footnotetext{
${ }^{6}$ The simulation results recorded for higher $\beta_{0}$ values result in improved EXIT characteristics, hence they were omitted from Figure $5 b$.

${ }^{7}$ This idealized simplifying assumption will be eliminated in the next section.
} 


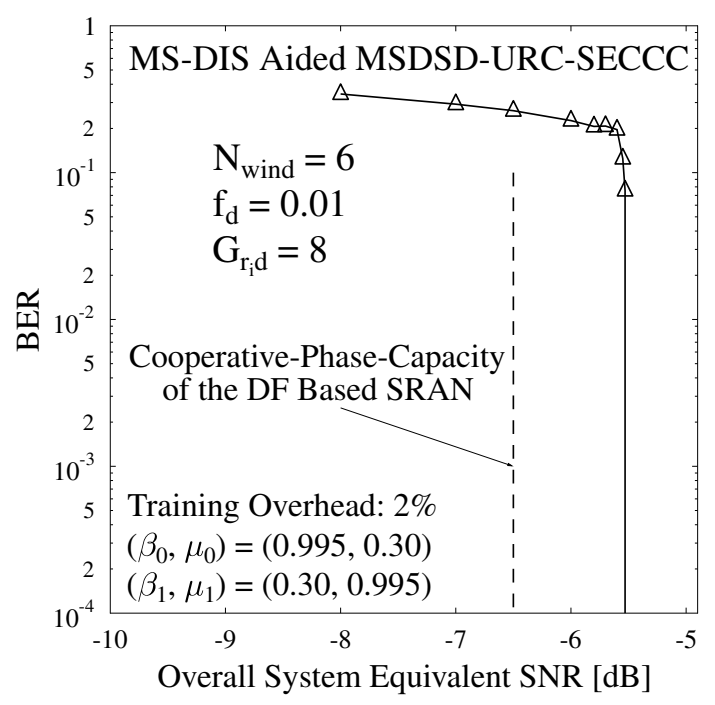

Fig. 7: The BER vs SNR performance of the BS receiver, corresponding to Figure 6.

When appropriately configuring the SECCC code [19] and the forgetting factors, the Monte-Carlo simulation based EXIT-trajectory of the MS-DIS aided MSDSD-URC-SECCC BS receiver is capable of approaching the point of perfect convergence at $(1,1)$ in Figure 6 after $I_{\text {outer }}^{d}=9$ outer iterations, when the SNR is as low as $-5.5 \mathrm{~dB}$. It is verified again in Figure 7 that our transceiver is capable of reliably operating at $-5.5 \mathrm{~dB}$, where the BER is shown to rapidly drop to an infinitesimally low value. Moreover, the corresponding capacity results of Figure 3 can be directly applied to Figure 7 as a benchmark, since the same system parameters are used. It is demonstrated in Figure 7 that the proposed BS receiver of Figure 2 attains a performance within about $1.0 \mathrm{~dB}$ of the DF based SRAN's capacity. A $2 \%$ training overhead is required for adapting the MS-DIS filter in the time-selective fading channel associated with a normalized Doppler frequency of $f_{d}=0.01$.

\section{Error Correction Performance at the RN}

The idealized simplifying assumption made in Section VI-C, namely that the relay perfectly decodes the SN's transmitted signals is verified with the aid of Figure 8. Owing to the compact dimensions of the mobile, the number of relay receiver antennas is fixed to $N_{r}=1$. Observe in Figure 8 that the SNR value has to be above $0.55 \mathrm{~dB}$ in order to allow the decoding trajectory of the proposed single-path MSDSDURC-SECCC relay decoder to approach the point of perfect convergence at $(1,1)$ in the EXIT Chart, even when assuming that the RN does not incur any interference. This implies that the error correction capability of our BS receiver may be significantly impaired by the potential error propagation encountered at the RN. Hence the system operating in a realistic interference-infested scenario is unable to exhibit an infinitesimally low BER at SNRs below $-5.5 \mathrm{~dB}$. However, the error correction capability of the RN receiver can be improved by introducing the cooperative-user-selection scheme of [29], or by sophisticated power-allocation and code-rate-

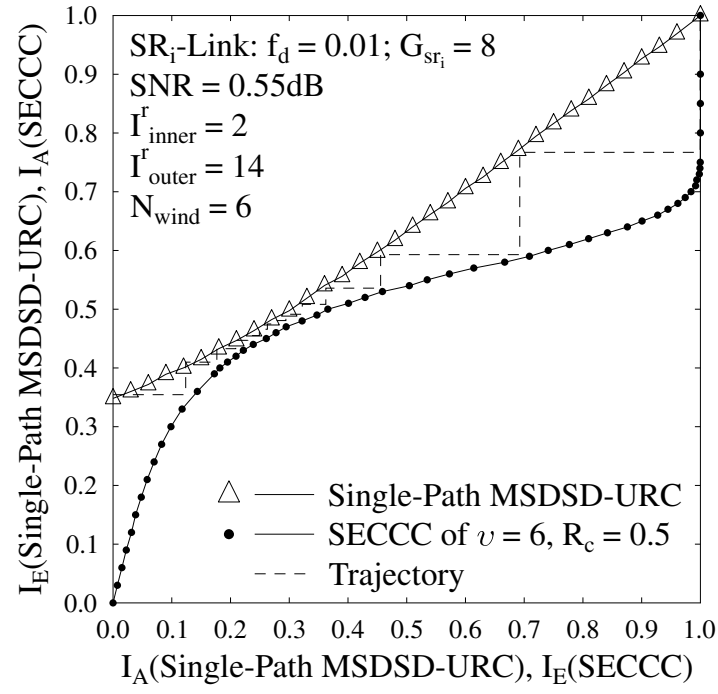

Fig. 8: EXIT chart characteristics at the RN.

optimization schemes [30], which will be addressed in our future research.

\section{CONCLUSIONS}

A novel adaptive Newton algorithm based MS-DIS filter was incorporated in the SRAN considered for the suppression of the successive relaying induced interference. The carefully designed MS-DIS aided MSDSD-URC-SECCC BS receiver exhibited a powerful error correction capability, and a vanishingly low BER for SNR values above $-5.5 \mathrm{~dB}$, while requiring as low as $2 \%$ training overhead. These contributions improved the practicality of our low-complexity noncoherent detection based SRAN. The relay receiver's poor error correction ability and the issues of inter-relay interference suppression remain open problems set aside for our future research.

\section{REFERENCES}

[1] P. Ho and D. Fung, "Error performance of multiple symbol differential detection of PSK signals transmitted over correlated Rayleigh fading channels," IEEE Trans. Commun., vol. 40, pp. 25-29, Oct. 1992.

[2] L. Lampe, R. Schober, V. Pauli, and C. Windpassinger, "Multiplesymbol differential sphere decoding," IEEE Trans. Commun., vol. 12, pp. 1981-1985, Dec. 2005.

[3] V. Pauli, L. Lampe, and R. Schober, “ 'Turbo DPSK' using soft multiplesymbol differential sphere decoding," IEEE Trans. Inf. Theory, vol. 52, pp. 1385-1398, Apr. 2006.

[4] L. Wang and L. Hanzo, "The amplify-and-forward cooperative uplink using multiple-symbol differential sphere-detection," IEEE Signal Process. Lett., vol. 16, pp. 913-916, Oct. 2009.

[5] L. Li and L. Hanzo, "Multiple-symbol differential sphere detection aided successive relaying in the cooperative DS-CDMA uplink," in Proc. 2011 IEEE WCNC, pp. 1875-1880.

[6] G. J. Foschini and M. J. Gans, "On limits of wireless communications in a fading environment when using multiple antennas," Wireless Personal Commun., vol. 6, pp. 311-335, Mar. 1998.

[7] E. C. Van der Meulen, "Three-terminal communication channels," $A d v$. Appl. Probability, vol. 3, pp. 120-154, 1971.

[8] Y. Fan, C. Wang, J. Thompson, and H. V. Poor, "Recovering multiplexing loss through successive relaying using repetition coding," IEEE Trans. Commun., vol. 6, pp. 4484-4493, Dec. 2007.

[9] C. Luo, Y. Gong, and F.-C. Zheng, "Interference cancellation in twopath successive relay system with network coding," in Proc. 2010 IEEE PIMRC, pp. 465-469. 
[10] C. Luo, Y. Gong, and F. Zheng, "Full interference cancellation for twopath relay cooperative networks," IEEE Trans. Veh. Technol., vol. 60, pp. 343-347, Jan. 2011.

[11] S. K. Cheung and R. Schober, "Differential spatial multiplexing," IEEE Trans. Wireless Commun., vol. 5, pp. 2127-2135, Aug. 2006.

[12] L. Wang and L. Hanzo, "Multiple-symbol detection aided differential spatial division multiple access," in Proc. 2011 IEEE ICC, pp. 1-5.

[13] J. Yang, F. Yang, H. S. Xi, and W. Guo, "Robust adaptive modified Newton algorithm for generalized eigendecomposition and its application," EURASIP J. Adv. Signal Process., vol. 2007, pp. 1-10, June 2007.

[14] L. K. Kong, S. X. Ng, R. G. Maunder, and L. Hanzo, "Near-capacity cooperative space-time coding employing irregular design and successive relaying," IEEE Trans. Commun., vol. 58, pp. 2232-2241, Aug. 2010.

[15] H. Ochiai, P. Mitran, and V. Tarokh, "Design and analysis of collaborative diversity protocols for wireless sensor networks," in Proc. IEEE VTC - Fall, vol. 7, pp. 4645-4649.

[16] H.-J. Su and E. Geraniotis, "Maximum signal-to-noise ratio array processing for space-time coded systems," IEEE Trans. Commun., vol. 50, pp. 1419-1422, Sep. 2002.

[17] S. Haykin, Adaptive Filter Theory, 3rd edition. Prentice-Hall, 1996.

[18] L. Li, L. Wang, and L. Hanzo, "The capacity of successive DF relaying and using soft multiple-symbol differential sphere detection," in Proc. 2011 IEEE GLOBECOM, pp. 1-5.

[19] M. F. Butt, R. Riaz, S. X. Ng, and L. Hanzo, "Near-capacity iterative decoding of binary self-concatenated codes using soft decision demapping and 3-D EXIT charts," IEEE Trans. Wireless Commun., vol. 9, pp. 1608-1616, May 2010.

[20] S. Benedetto, D. Divsalar, G. Montorsi, and F. Pollara, "Selfconcatenated trellis coded modulation with self-iterative decoding," in Proc. 1998 IEEE GLOBECOM, vol. 1, pp. 585-591.

[21] D. Divsalar, S. Dolinar, and F. Pollara, "Serial concatenated trellis coded modulation with rate-1 inner code," in Proc. 2000 IEEE GLOBECOM, vol. 2, pp. 777-782.

[22] L. Hanzo, R. Maunder, J. Wang, and L. L. Yang, Near-Capacity Variable-Length Coding: Regular and Exit-Chart Aided Irregular Designs. John Wiley \& Sons, 2011.

[23] C. Douillard, A. Picart, P. Didier, M. Jezequel, C. Berrou, and A. Glavieux, "Iterative correction of intersymbol interference: turboequalization," Euro. Trans. Telecommun., vol. 6, pp. 507-511, Sep. 1995.

[24] L. Hanzo, T. H. Liew, B. L. Yeap, and R. Y. S. Tee, Turbo Coding, Turbo Equalisation and Space-Time Coding: EXIT-Chart Aided NearCapacity Designs for Wireless Channels. John Wiley \& Sons and IEEE Press, 2011.

[25] A. Hst-Madsen and J. Zhang, "Capacity bounds and power allocation for wireless relay channels," IEEE Trans. Inf. Theory, vol. 51, pp. 20202040, June 2005.

[26] R. Schober and L. Lampe, "Noncoherent receivers for differential spacetime modulation," IEEE Trans. Commun., vol. 50, pp. 768-777, May 2002.

[27] L. Wang, L. Kong, S. X. Eg, and L. Hanzo, "To cooperate or not: a capacity perspective," in Proc. 2010 IEEE VTC - Spring, pp. 1-5.

[28] I. Land, S. Huettinger, P. A. Hoeher, and J. B. Huber, "Bounds on information combining," IEEE Trans. Inf. Theory, vol. 51, pp. 612-619, Feb. 2005 .

[29] E. Beres and R. Adve, "Selection cooperation in multi-source cooperative networks," IEEE Trans. Wireless Commun., vol. 7, pp. 118-127, Jan. 2008.

[30] L. Hanzo, Y. Akhtman, M. Jiang, and L. Wang, MIMO-OFDM for LTE, WIFI and WIMAX: Coherent versus Non-Coherent and Cooperative Turbo-Transceivers. John Wiley \& Sons and IEEE Press, 2010.

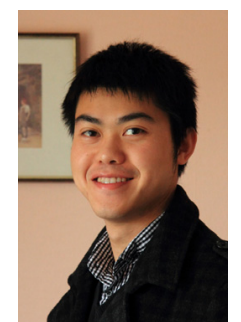

Li $\mathbf{L i}$ received the B.Eng. degree in information engineering from the University of Electronic Science and Technology of China (UESTC), Chengdu, China, in 2006 and the M.Sc. degree with distinction in wireless communications from the University of Southampton, Southampton, U.K., in 2009. He is currently working towards the Ph.D. degree in the Communications Group, School of Electronics and Computer Science, University of Southampton, Southampton, U.K., and participating in the European Union Concerto project. His research interests include channel coding, iterative detection, non-coherent transmission technologies, cooperative communications and network coding.

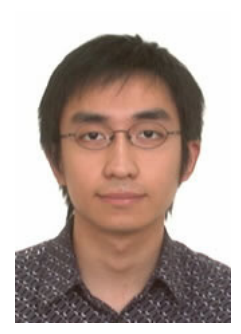

Li Wang (S'09-M'10) was born in Chengdu, China, in 1982. He received his B.Eng. degree in information engineering from Chengdu University of Technology (CDUT), Chengdu, China, in 2005 and his M.Sc. degree with distinction in radio frequency communication systems from the University of Southampton, UK, in 2006. Between October 2006 and January 2010, he was a PhD student in the Communications Group, School of Electronics and Computer Science, University of Southampton, and participated in the Delivery Efficiency Core Research Programme of the Virtual Centre of Excellence in Mobile and Personal Communications (Mobile VCE). He was awarded a joint EPSRC and Mobile VCE full scholarship. Upon the completion of his Ph.D. in January 2010, he conducted research as a senior research fellow in School of Electronics and Computer Science at the University of Southampton, and has been involved in Project \#7 of the Indian-UK Advanced Technology Centre (IU-ATC): Advanced air interface technique for MIMO-OFDM and cooperative communications. His research interests include space-time processing, channel coding, multi-user detection, non-coherent transmission techniques, iterative detection, EXIT-chart-aided system design, MIMO-OFDM and cooperative systems.

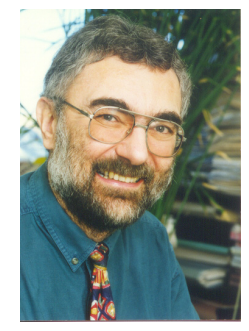

Lajos Hanzo FREng, FIEEE, FIET, Fellow of EURASIP, DSc received his degree in electronics in 1976 and his doctorate in 1983. In 2009 he was awarded the honorary doctorate "Doctor Honoris Causa" by the Technical University of Budapest. During his 35-year career in telecommunications he has held various research and academic posts in Hungary, Germany and the UK. Since 1986 he has been with the School of Electronics and Computer Science, University of Southampton, UK, where he holds the chair in telecommunications. He has successfully supervised about $80 \mathrm{PhD}$ students, co-authored 20 John Wiley/IEEE Press books on mobile radio communications totalling in excess of 10,000 pages, published $1250+$ research entries at IEEE Xplore, acted both as TPC and General Chair of IEEE conferences, presented keynote lectures and has been awarded a number of distinctions. Currently he is directing an academic research team, working on a range of research projects in the field of wireless multimedia communications sponsored by industry, the Engineering and Physical Sciences Research Council (EPSRC) UK, the European IST Programme and the Mobile Virtual Centre of Excellence (VCE), UK. He is an enthusiastic supporter of industrial and academic liaison and he offers a range of industrial courses. He is also a Governor of the IEEE VTS. Since 2008 he has been the Editor-in-Chief of the IEEE Press and since 2009 a Chaired Professor also at Tsinghua University, Beijing. For further information on research in progress and associated publications please refer to http://wwwmobile.ecs.soton.ac.uk 